\title{
Amaryllidaceae: Fuente Potencial de Alcaloides. Actividades Biológicas y Farmacológicas
}

ISSN 0122-8420 elSSN2539-0899

Fecha de recepción: 07 de julio de 2020

Fechadeaceptación: 27 de agosto de 2020

DOI: https://doi.org/10.19053/01228420.v17.n3.2020.11379
Carlos Felipe González-Chavarro • 1 Marco Cabezas Gutierrez • 2 Víctor Camilo Pulido-Blanco . 3 Xiomara Melissa Celis Ruiz . 4

\section{Resumen}

Las plantas de la familia Amaryllidaceae han adquirido importancia por ser utilizadas como ornamentales, por tener flores y apariencia vistosa, además de sus amplias propiedades farmacológicas. Los géneros principalmente estudiados son Amaryllis, Crinum, Galanthus, Narcisus, Bruganvigia y Lycoris. Ellos producen alcaloides teniendo como precursores primarios la L-fenilalanina y L-tirosina, los cuales dan origen al principal esqueleto carbonado denominado norbelladina, que a través de la oxidación del acoplamiento del fenol genera estructuras con posiciones de tipo orto - para', para - orto' y para - para', agrupando los tipos de alcaloides isoquinólicos, tales como licorina, homolicorina, crinina, haemantamina, tazetina, narciclasina, montanina y galantamina. Estos alcaloides tienen actividad antiviral y antimicrobiana; son citotóxicos, anticonvulsivos, antitumorales, hipotensivos y antiinflamatorios. El alcaloide primordial de esta familia es la galantamina, la cual es producida a partir de las plantas Leucojum sativum o Narcissus pseudonarcissus, y utilizada terapéuticamente para inhibir la actividad de la acetilcolinesterasa, contrarrestando los síntomas de las afecciones del sistema nervioso causadas por la enfermedad de Alzheimer.

Palabras clave: metabolismo secundario, isoquinólicos, galantamina, fitoquímica, Alzheimer.

\section{Amaryllidaceae: Potential Sourse of Alkaloids. Biological and Pharmacological Activities}

\begin{abstract}
Plants of Amaryllidaceae family have gained importance for being used as ornamentals, for having flowers and a showy appearance, in addition to their extensive pharmacological properties. The genera mainly studied are Amaryllis, Crinum, Galanthus, Narcisus, Bruganvigia and Lycoris. They produce alkaloids having as primary precursors L-phenylalanine and L-Tyrosine, which give rise to the main carbon skeleton called norbelladine, which through oxidation of the coupling of phenol, generates structures with ortho-para 'type positions, for - ortho 'and para - para', grouping the types of isoquinolic alkaloids, such as liquorine, homolicorin, crinine, haemantamine, tazetine, narcyclasin, montanin and galantamine. These alkaloids have antiviral, antimicrobial, cytotoxic, anticonvulsant, antitumor, hypotensive, and anti-inflammatory activity. The main alkaloid of this family is galantamine, being produced from the plants Leucojum sativum or Narcissus pseudonarcissus, and used therapeutically to inhibit the activity of acetylcholinesterase, counteracting the symptoms caused by the damage of the nervous system by Alzheimer's disease.
\end{abstract}

Keywords: secondary metabolism, isoquinolines, galantamine, phytochemistry, Alzheimer's disease.

I. MSc. Corporación Colombiana de InvestigacióAgrapecuaria Agrasavia. Centra de Investigación La Libertad, Villavicencio, Meta. Autar para correspandencia:

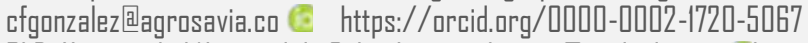

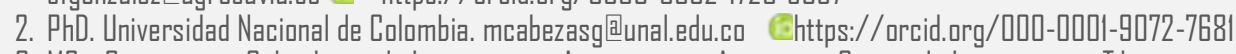

3. MSc. Corporación Colombiana de Investigación Agropecuaria Agrasavia. Centro de Investigación Tibaitatá, sede Tunja, Bayacá. vpulida@agrasavia.co

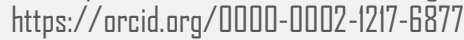

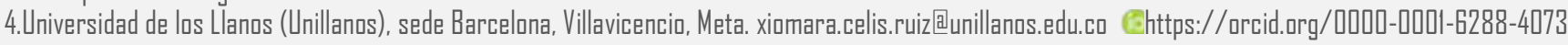




\section{I.Introducción}

El hombre a lo largo de los años seleccionó apenas cerca de 300 vegetales para su alimentación, y a partir de un poco más de 100 obtuvo principios activos puros para el tratamiento de enfermedades (Pinto et al., 2002). Las plantas, como parte de la alimentación o en preparaciones medicinales, han sido usadas para curar o prevenir enfermedades a lo largo de la historia. Fósiles indican que hace 60000 años los vegetales ya eran usados por el hombre para fines medicinales. Actualmente, las plantas constituyen una parte integral de las actividades terapéuticas de casi el $65 \%$ de la población mundial (Elgorashi et al., 2003). Entre estas fuentes tradicionales de medicamentos, las monocotiledóneas de la familia Amaryllidaceae comprenden cerca de 85 géneros y más de 1100 especies, con amplia distribución geográfica, presentando tres principales centros de diversidad: América del Sur, África del Sur y la zona mediterránea de Europa (Arroyo \& Cutler, 1984; Meerow, 2004; Unver, 2007). Sus representantes se caracterizan por ser hierbas bulbosas (raramente rizomatosas), con hojas dísticas, inflorescencia en forma de umbela (unifloras o plurifloras), flores bisexuales y ovarios ínferos (Meerow, 2004). Debido a lo llamativo de sus flores, las especies de esta familia son utilizadas en muchos países como ornamentales (Amaryllis, Narciso y Galanthus); además, esta familia está considerada como una de las 20 más importantes que presentan alcaloides en su composición (Unver, 2007; Jin, 2013).

Los alcaloides de Amaryllidaceae (AA), que son específicos para esta familia de plantas, fueron inicialmente reconocidos en el siglo XIX con el aislamiento de licorina de Narcissus pseudonarcissus. En 1950 fueron retomados con mayor intensidad (Ghosal et al., 1988), y desde entonces han sido aislados más de 500 AA estructuralmente diversos (Jin, 2013). Los alcaloides son compuestos heterocíclicos nitrogenados derivados principalmente de aminoácidos, y en la naturaleza se pueden encontrar como sales con el ácido acético, láctico, málico, tartárico, cítrico y oxálico (Arango, 2008). Son de vital importancia en la célula, ya que cumplen un papel muy importante en el mimetismo hormonal de la planta y son necesarios para el metabolismo. Aunque sus estructuras difieran mucho entre unas y otras, recientes estudios fitoquímicos han demostrado la diversidad de sus actividades farmacológicas en los numerosos miembros de AA. Estas incluyen analgésica, anticancerígena y actividades antimicrobianas (Bastida et al., 2011; Heinrich \& Lee Teoh, 2004; Kornienko \& Evidente, 2008; Nair \& Van Staden, 2013; Nair \& Van Standen, 2019; Nair \& Van Standen, 2020a; Nair \& Van Standen, 2020b).

En las plantas, dichos metabolitos cumplen un papel de almacenamiento de nitrógeno, sirven como sustancias protectoras de los frutos, semillas, corteza del tallo, raíz y epidermis de las hojas (Vélez-Terranova et al, 2014). Además son factores importantes que ayudan al desarrollo de la planta, pero no son indispensables en algunas de ellas, ya que aquellas que no producen estos metabolitos, tienen otros que ayudan a su desarrollo (Vélez-Terranova et al., 2014). Este trabajo revisa el estado del conocimiento de los alcaloides en Amaryllidaceae, los géneros productores, la biosíntesis y algunas de sus propiedades biológicas y farmacológicas.

\section{Algunos Géneros De Interés}

De los 85 géneros presentes, los principales estudios se han desarrollado en Amaryllis, Crinum, Hymenocallis, Hippeastrum, Haemanthus y Zephyranthes, (Tabla 1). Algunas especies de estos géneros producen fácilmente híbridos, por lo cual existen dificultades en su taxonomía (Tram et al., 2002). 
Tabla 1. Estructuras de alcaloides presentes en los géneros Amaryllis, Crinum, Hymenocallis, Hippeastrum, Haemanthus y Zephyranthes de la familia Amaryllidaceae

\begin{tabular}{|c|c|c|}
\hline $\begin{array}{l}\text { Género en el cual está } \\
\text { presente }\end{array}$ & Nombre & Estructura del Alcaloide \\
\hline Crinum, Hymenocallis & Crinina & \\
\hline Amaryllis, Hymenocallis & Galantamina & \\
\hline Haemanthus & Licorina & \\
\hline $\begin{array}{c}\text { Amaryllis, } \\
\text { Crinum, Hippeastrum, } \\
\text { Hymenocalis y Zephyranthes }\end{array}$ & Homocolina & \\
\hline Haemanthus y Hymenocallis & Pancracina & \\
\hline \multirow{3}{*}{ Crinum } & Cripovelina & \\
\hline & Augustamina & \\
\hline & Beladina & \\
\hline $\begin{array}{c}\text { Crinum, Hippeastrum y } \\
\text { Hymenocallis }\end{array}$ & Tazetina & \\
\hline $\begin{array}{l}\text { Crinum, Hippeastrum, } \\
\text { Hymenocallis y Zephyranthes }\end{array}$ & $\begin{array}{l}\text { Pancrastistanin } \\
\text { a }\end{array}$ & \\
\hline
\end{tabular}

Fuente: tomado y modificado de Sousa da Silva (2009) y Rojas-Vera et al. (2020). 


\section{A. Género Amaryllis}

Son conocidas por ser usadas como ornamentales, reservar alcaloides y para el tratamiento de enfermedades. La especie principal es Amaryllis belladonna, y ha sido de gran interés ya que se ha evidenciado que posee actividad contra la leucemia por su fracción del alcaloide tipo licorina (Pettit et al., 1984; Rojas-Vera et al., 2019; Shammari et al., 2020). En América, de este género se han logrado aislar alcaloides como anidrolicorina, ambelina, undulatina y clorhidrato de anidrolicorina, que también han sido de interés para el tratamiento de esta enfermedad (Evidente et al., 2004; Shammari et al., 2020).

\section{B. Género Crinum}

Comprende cerca de 160 especies distribuidas en las regiones tropicales de Asia, Australia, África y América. Las especies de este género poseen flores muy llamativas que son usadas para ornamentación (Tram et al., 2002).

De este género se han logrado aislar alcaloides de tipo crinina (también conocidos como 5,10b-etanofenatridina) y tipo licorina. Algunas especies de las que se han aislado alcaloides son: C. asiatum, C. americanum, C. bulbispermum, C. latifolium, C. macowanii, C. amabile, C. erubescence y C. augustum (Tram et al., 2002). Estos dos tipos de alcaloides difieren entre sí, principalmente en la cantidad y en la posición de los enlaces dobles localizados en los anillos C y D y en el tipo de los sustituyentes en el anillo $C$, tanto en su posición como estereoquímica (Tram et al., 2002).

Para el género, una de las especies de mayor importancia es la C. elagoense, especie ampliamente estudiada para su uso en el tratamiento de pacientes con cáncer, especialmente en zonas como África del Sur (Nair \& Staden, 2020b).

\section{Género Hymenocallis}

El primer alcaloide fue aislado del género Hymenocallis en 1970 (Rivero et al., 2004), de la especie Hymenocallis expansa, de la cual se aislaron tres alcaloides (tazetina, hipeastrina y haemantridina), que presentan actividad citotóxica contra células humanas (Antoun et al., 1993).

En 1993 fueron aislados los alcaloides pancratistatina, narciclasina y 7-desoxi-narciclasina de la especie Hymenocallis littoralis Salisb. Presentó actividad antineoplásica contra sarcomas, melanomas y virus (Rivero et al., 2004; Ivanov et al., 2012).

\section{Género Hippeastrum}

Debido a su parecido en cuanto a caracteres morfológicos, las especies de este género suelen ser confundidas con las del género Amaryllis; sin embargo, la taxonomía de Bureau da claridad en sus diferencias taxonómicas por su distribución, ya que Amaryllis es africano y Hippeastrum es americano (Dutilh, 1996). Dentro de los principales alcaloides encontrados en este género están la licorina, pancracina, montaina, vitatina, tazetina y pretazetina.

\section{E. Género Haemanthus}

Es un género pequeño con 22 especies nativas de Sudáfrica. Su principal característica es la inflorescencia, lo que las hace muy populares para su uso ornamental (Nair \& Van Staden, 2020b). 
Especies de este género, al igual que las del género Hymenocallis, han sido de gran interés para el tratamiento de personas con cáncer. Un ejemplo de lo anterior es la especie Haemanthus humilis, donde se ha comprobado que posee agentes antiproliferativos contra algunas líneas celulares de cáncer humano (Nair \& Van Staden, 2020a; Nair \& Van Staden, 2020b).

\section{F. Género Zephyranthes}

Originario de América tropical y subtropical, las especies de Zephytanthes son encontradas principalmente en países como Colombia, en regiones de Cundinamarca, Boyacá y Antioquia (Fernández \& Groenendijk, 2004). Se ha reportado la presencia de alcaloides como crinina, licorina y galantamina entre otros (Herrera et al., 2001). En el caso de Z. concolor y Z. robusta hay evidencia de la biosíntesis de galantamina (Reyes-Chilpa et al., 2011). En el caso de Z. carinata, ya se han registrado alcaloides como licorina, galantina y haemantamina; alcaloides de interés para el tratamiento de la leucemia (Cahlíková et al., 2011).

\section{Clasificación de los alcaloides}

Los alcaloides se han clasificado en función de su estructura. Se distinguen los compuestos heterocíclicos de los no heterocíclicos, lo que en la actualidad permite clasificarlos de varias formas (Paris \& Hurabielle, 1981; Bruneton, 1987; Arango, 2008):

De acuerdo con sus propiedades farmacológicas:

- Modificadores del sistema nervioso central (estimulantes nerviosos): alcaloides de la iboga: iboganina; alcaloides de la nuez vómica: estricnina.

Alucinógenos: alcaloides del peyote: mescalina; alcaloides del yagé: harmalina.

- $\quad$ Modificadores del sistema nervioso autónomo (parasintopatomiméticos): de acción directa como jaborandi: pilocarpina, o anticolinesterásicos como habas de calabar: eserina.

Parasintopatolíticos: belladona, que produce la atropina; efedras, que producen la efedrina.

De acuerdo con su distribución botánica:

- $\quad$ Alcaloides del tabaco: nicotina; alcaloides de las Solanaceae midriáticas: atropina hiosciamina; alcaloides de Amarillidaceae.

De acuerdo con su origen biosintético:

Alcaloides derivados de aminoácidos alifáticos: cocaína, lobelina.

Alcaloides derivados de aminoácidos aromáticos: morfina, boldina, ergotamina. 
En este contexto se adoptará la última forma de clasificación, puesto que muestra una gran diversidad estructural y homogeneidad bioquímica; es decir, se pueden agrupar todos los alcaloides naturales conocidos por ser originados a partir de un restringido número de aminoácidos o de precursores biogenéticos. Esta aproximación biogenética es indispensable como ayuda quimiotaxonómica y está aplicada especialmente para la química estructural en la asignación de posiciones oxigenadas y para las síntesis biomimética de sustancias (Arango, 2008).

Se pueden distinguir (Figura 1):

- Alcaloides alifáticos: derivados de la ornitina (pirrolidinas, tropánicos, pirrolizidínicos), derivados de la lisina (piperidinas, quinolizidínicos).

-Alcaloides aromáticos: derivados del ácido nicotínico (piridinas), derivados de la fenilalanina y tirosina (isoquinoleinas), derivados del triptófano (indólicos, quinoleínas), derivados del ácido antranílico (quinoleínas) y derivados de la histidina (imidazoles).

- Alcaloides de origen diverso: alcaloides terpénicos y esteroidales, alcaloides diversos (purinas, macrociclos)
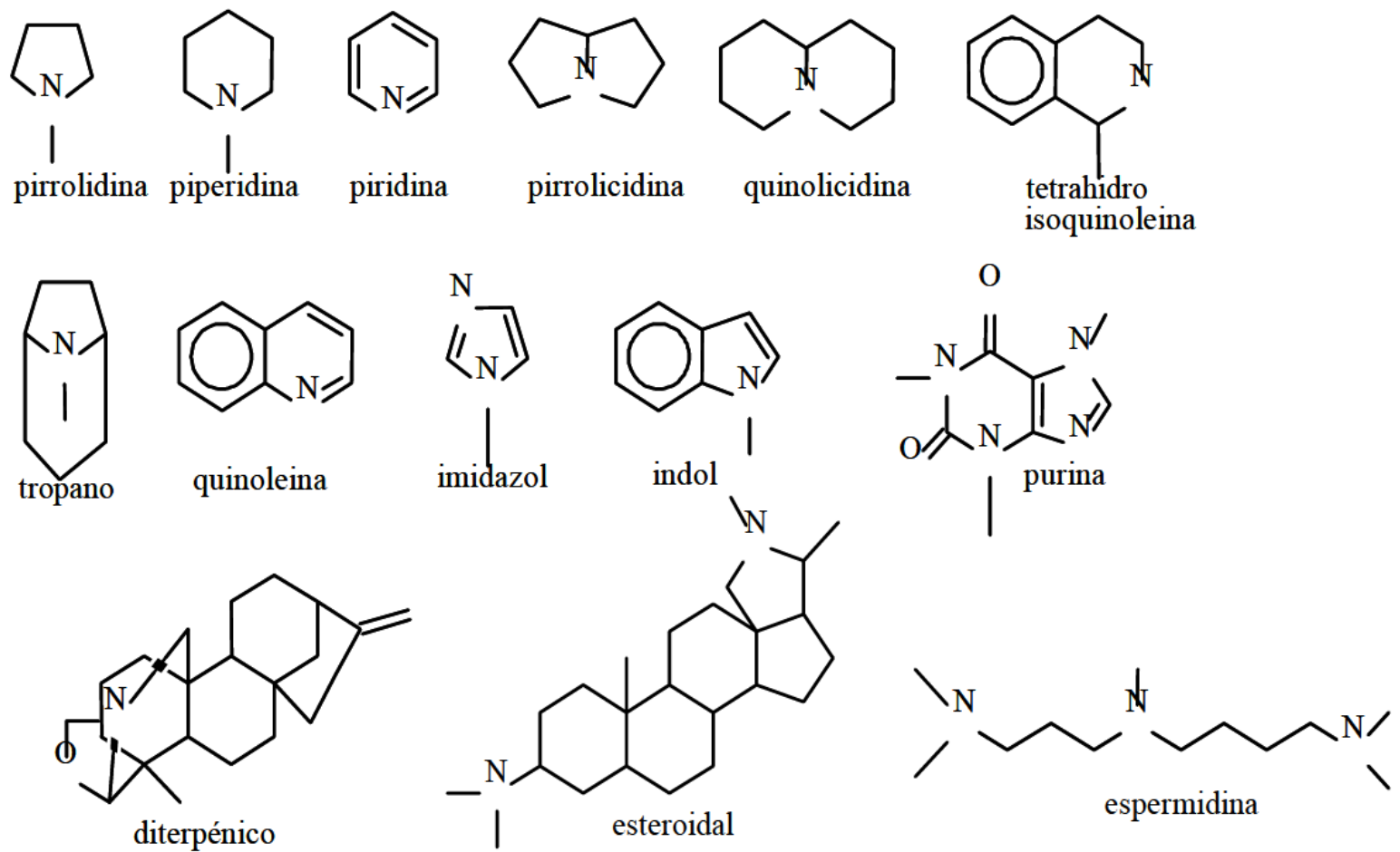

Figura 1. Algunos núcleos alcaloidicos.

Fuente: Arango (2008).

\section{Biosíntesis de alcaloides}

La mayor parte de la investigación de biosíntesis de alcaloides de Amaryllidaceae se ha llevado a cabo en los años sesenta y principios de los setenta (Cedrón, 2010). Desde entonces, el estudio ha sido enfocado en la biosíntesis de galantamina y alcaloides relacionados (Eichhorn et al.,1998). El amplio número de estos compuestos y las múltiples variaciones en su estructura que presentan los alcaloides de esta familia se deben a las rutas biosintéticas que los producen, por lo que se encuentran clasificados nueve esqueletos del tipo: norbelladina, licorina, 
homolicorina, crinina, hemantamina, narciclasina, tazetina, montanina y galantamina (Bastida et al., 2011). Estos alcaloides constituyen un subgrupo característico derivado de la tirosina y de la fenilalanina, los cuales se originan de un mismo precursor biosintético denominado norbelladina o beladina (Jin, 2013), el cual está formado por la condensación de aminoácidos aromáticos derivados de 3,4-dihidroxibenzaldehído (3,4-DHBA también llamado aldehído protocatéquico) y tiramina (Figura 2).

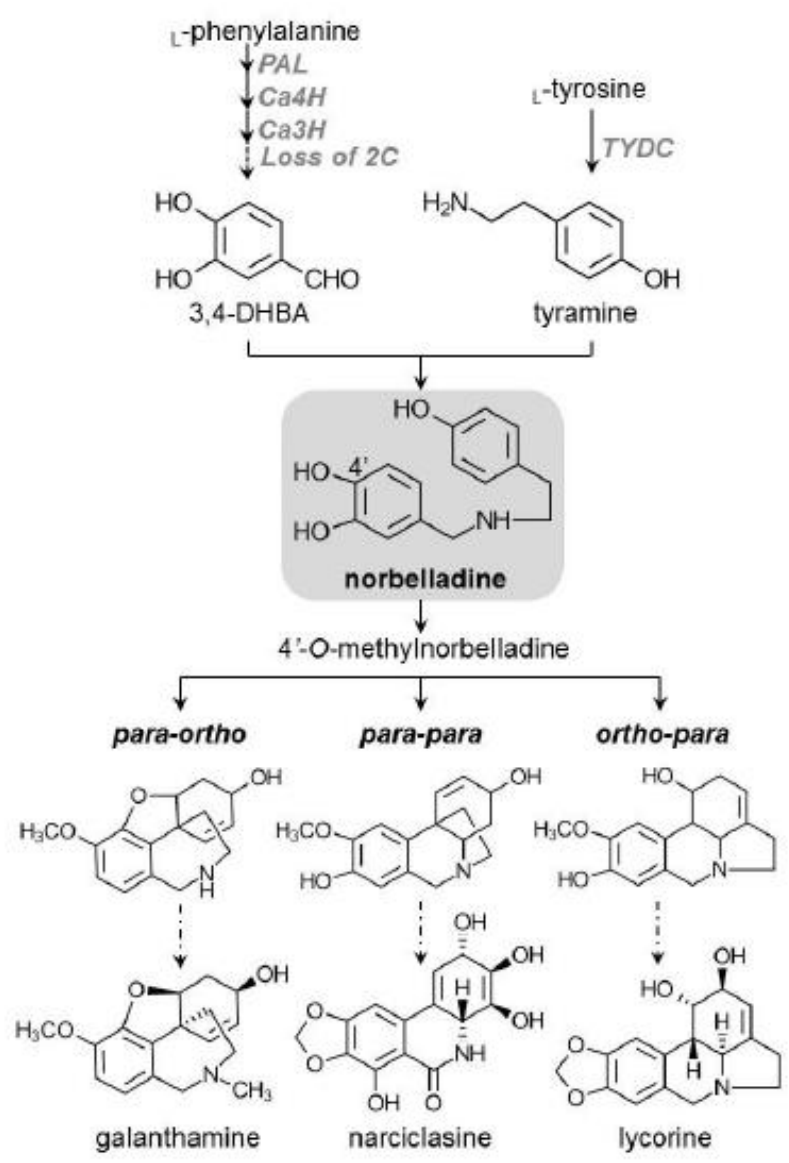

Figura 2. Vía de biosíntesis de alcaloides de Amaryllidaceae (AA).

Fuente: Singh y Desgagné-Penix (2014).

Los sustratos y las enzimas comprometidos en el metabolismo de la planta, a menudo han sido traídos desde el metabolismo primario, especialmente el aminoácido aromático L-fenilalanina y L-tirosina (Chu et al., 2011).

En las etapas iniciales de la biosíntesis de AA, la enzima fenilalanina amonio liasa (PAL) cataliza la eliminación de amoníaco para generar ácido transcinámico (Figura 2). Dos reacciones de hidroxilación catalizada por citocromo P450, cinamato-4-hidroxilasa $(\mathrm{CA} 4 \mathrm{H})$ y cumarato-3-hidroxilasa $(\mathrm{Ca} 3 \mathrm{H})$ seguidas por la pérdida de dos átomos de carbono, conducen a la formación del precursor C6C1, 3,4-DHBA (Bastida et al., 2011; Eichhorn et al., 1998; Grisebach, 1973; Osorio, 2004-2006). Cabe mencionar que estas reacciones son también parte del metabolismo de fenilpropanoides, por lo que la biosíntesis de AA muestra muy bien las conexiones entre las dos vías especializadas, alcaloide y fenilpropanoides.

Por otro lado, la tirosina se descarboxila a la tiramina por tirosina-descarboxilasa (TYDC) (Bastida et al., 2011; Eichhorn et al., 1998; Grisebach, 1973; Takos \& Rook, 2013). TYDC es una enzima reguladora clave única en muchas plantas productoras de alcaloides. Además de controlar la transición de la ruta primaria a la especializada, TYDC asegura un suministro adecuado de tiramina para la síntesis de diversos alcaloides de isoquinolina, 
incluyendo la bien conocida morfina y la codeína, analgésicos narcóticos (Desgagné-Penix et al., 2011; Facchini, 2001; Facchini et al., 2000; Hagel \& Facchini, 2013; Ziegler \& Facchini, 2008).

Todos los miembros de AA son vistos como derivados de la estructura común de esqueleto de norbelladina. La combinación de 3,4-DHBA y tiramina son resultados de la formación de una base de Schiff intermedia, que después se reduce a norbelladina y a O-metilnorbeladina (Dewick, 2009; Ghosal et al., 1988; Cedrón, 2010).

Luego se da la O-metilación de norbelladina, que precede al acoplamiento oxidativo de fenol. Esta característica da lugar a la amplia diversidad estructural de los miembros de AA. Las reacciones de 0-metilación de las plantas son la transformación común en la biosíntesis de alcaloides y están más a menudo catalizadas por la S-adenosil-Lmetionina (SAM) metiltransferasas dependientes (MTS) (Dewick, 2002; Liscombe et al., 2012). Por lo tanto, se supone que norbelladina debe ser de 4'-0-metilado para formar 4 'O metil norbelladina. Este compuesto sirve entonces como el intermediario central de las múltiples rutas biosintéticas para conducir a diversos tipos estructurales de los AA (Figura 2).

Un paso crucial en la biosíntesis de AA es la ciclación de 4 'O metil norbelladina por oxidación del acoplamiento C-C fenol, que genera tres estructuras de cadena principal que se conocen como posiciones de tipo orto - para', para orto' y para - para' (Figura 3) (Cedrón, 2010).

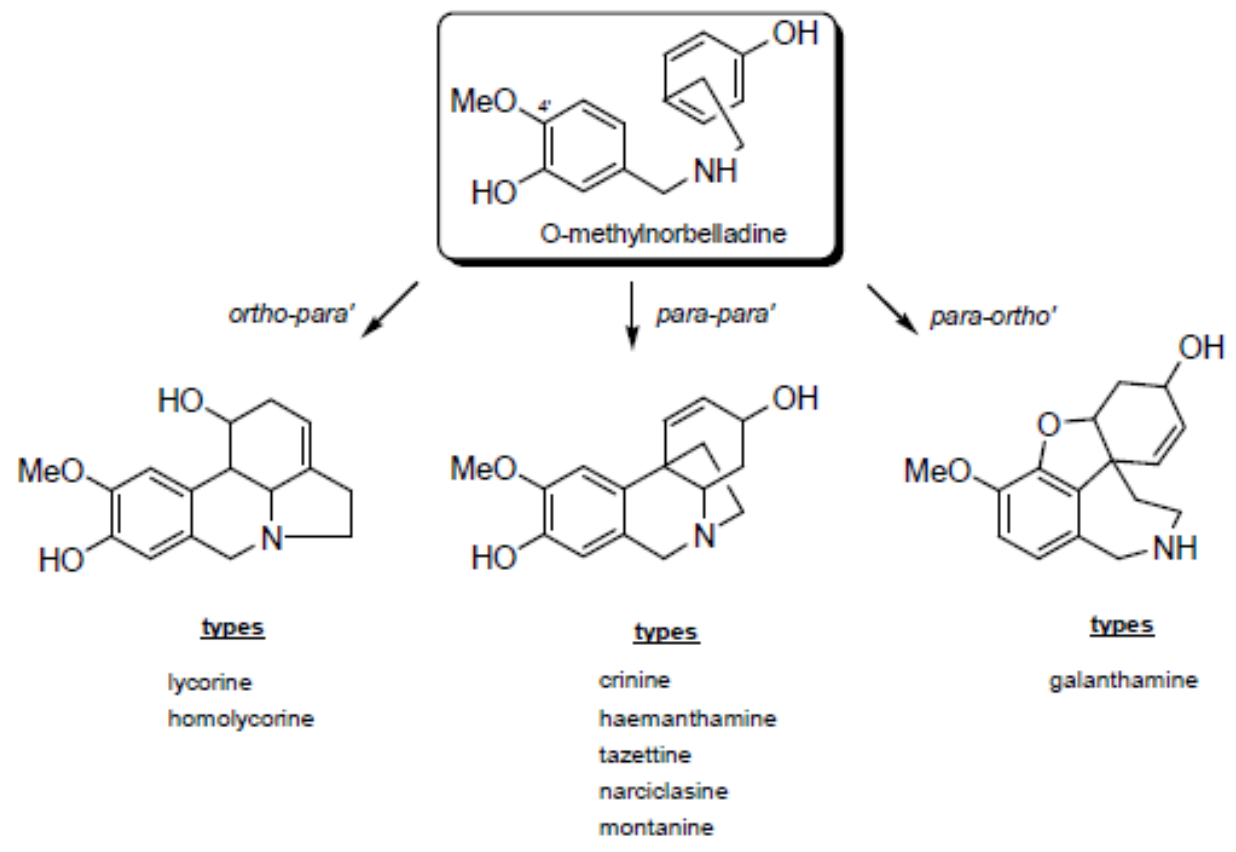

Figura 3. Acoplamiento fenol oxidativo en Amaryllidaceae.

Fuente: Bastida et al. (2011).

\section{A. Formación de licorina y homolicorina}

La licorina y homolicorina son alcaloides derivados del pirrol fenantridina (tipo licorina) y la 2-benzopirano-[3,4-g] indol (tipo homolicorina), los cuales se generan a partir de un acoplamiento fenol oxidativo orto-para (Jin, 2013). La conversión biológica de aldehído protocatéquico en licorina procede a través de 0-metil norbelladina y norpluviina. En primer lugar, implica una reducción del aldehído carbonilo, y después, en la generación de licorina, la oxidación de este mismo átomo de carbono. La estereoquímica absoluta de estos procesos ha sido elucidada en 
experimentos posteriores (Fuganti, 1973), y los resultados muestran que la adición de hidrógeno y remoción se llevan a cabo en la nueva cara de las moléculas implicadas, siendo el hidrógeno introducido inicialmente y luego eliminado (Fuganti \& Mazza, 1971). Es de destacar que norpluviina, a diferencia de pluviina, es convertida en Narcissus principalmente a los alcaloides de tipo homolicorina. La oxidación bencílica en la posición 6 , seguida de un anillo abierto, forma un aldehído amino; la formación de hemiacetal y la posterior metilación proporcionan la licorina (Harken et al., 1976), que después de la oxidación da homolicorina (Figura 4).

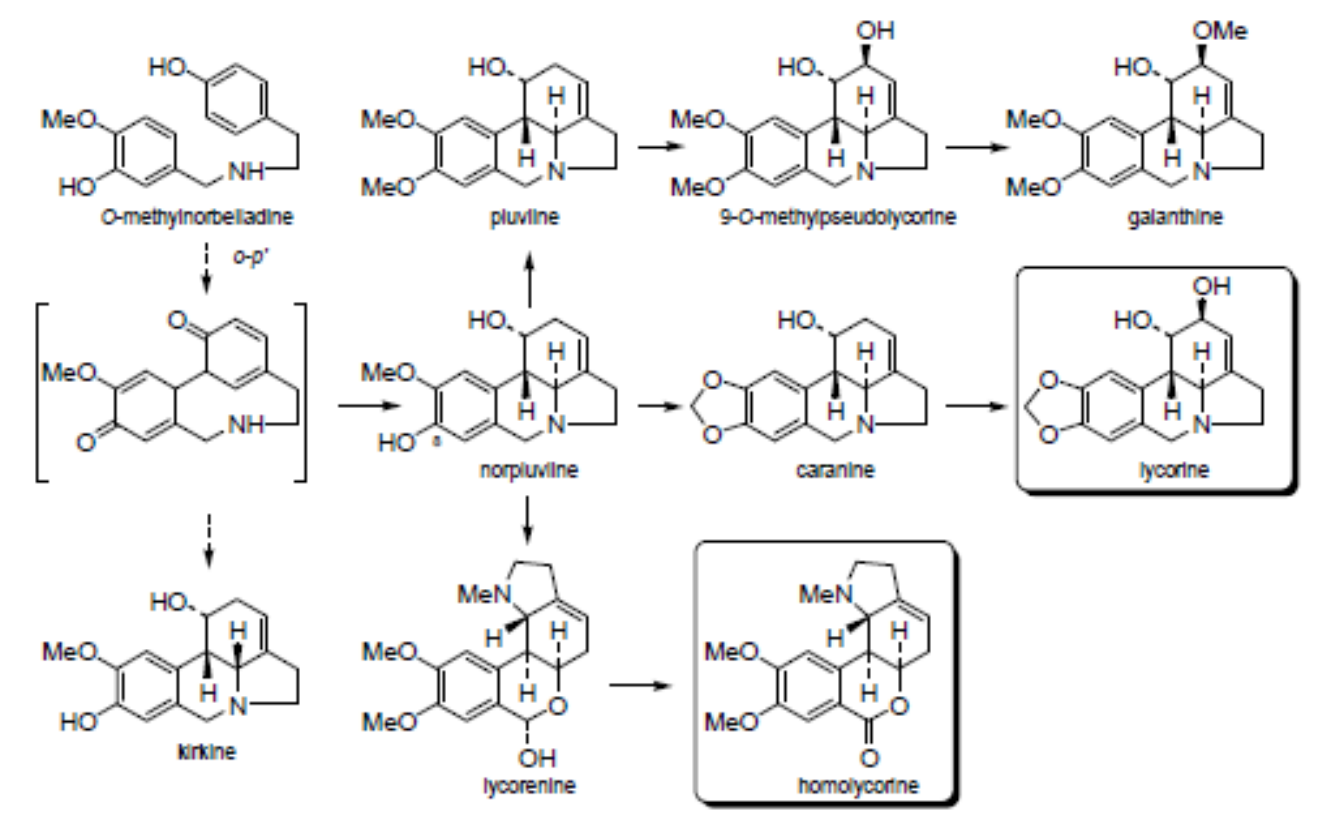

Figura 4. Alcaloides procedentes de un acoplamiento orto - para'.

Fuente: Bastida et al. (2011).

\section{B. Formación de tipo crinina, hemantamina, tazetina, narciclasina y montaina}

Este grupo incluye los alcaloides derivados de 5,10b-etano fenantridina (tipos crinina y hemantamina), 2benzopirano [3,4-c] indol (tipo tazetina), fenantridina (tipo narciclasina) y 5,11- metano morfantridina (tipo montanina), esqueletos procedentes de un acoplamiento fenol oxidativo para - para (Wildman \& Bailey, 1969).

La conversión de biosíntesis de la 5,10b-etano fenantridina a la 2-benzopirano [3,4-c] indol fue demostrado por la alimentación de alcaloides marcados con tritio a Sprekelia formosissima. Se comprobó que esta planta convierte a haemantamina haemantidina/epihaemantamina y posteriormente a pretazetina de una manera esencialmente irreversible (Fales \& Wildman, 1964). Esta transformación se considera que procede de un intermedio que nunca ha sido detectado por métodos espectrales (Wildman \& Bailey, 1969).

También se ha demostrado que el alcaloide narciclasina procede de la vía de la biosíntesis de alcaloides tipo crinina y tipo hemantamina, y no a través de derivados de norpluviina y licorina. De hecho, en vista de su afinidad estructural tanto con hemantamina como con licorina, narciclasina podría derivarse de otra vía. En un ensayo de marcación se obtuvo claramente que $\mathrm{O}$ - metil norbelladina está completamente retenido en los alcaloides mencionados, y proporciona un patrón interno satisfactorio; también el grado de retención de tritio es una guía confiable a la dirección de acoplamiento oxidativo fenol para - para' (Figura 5). 


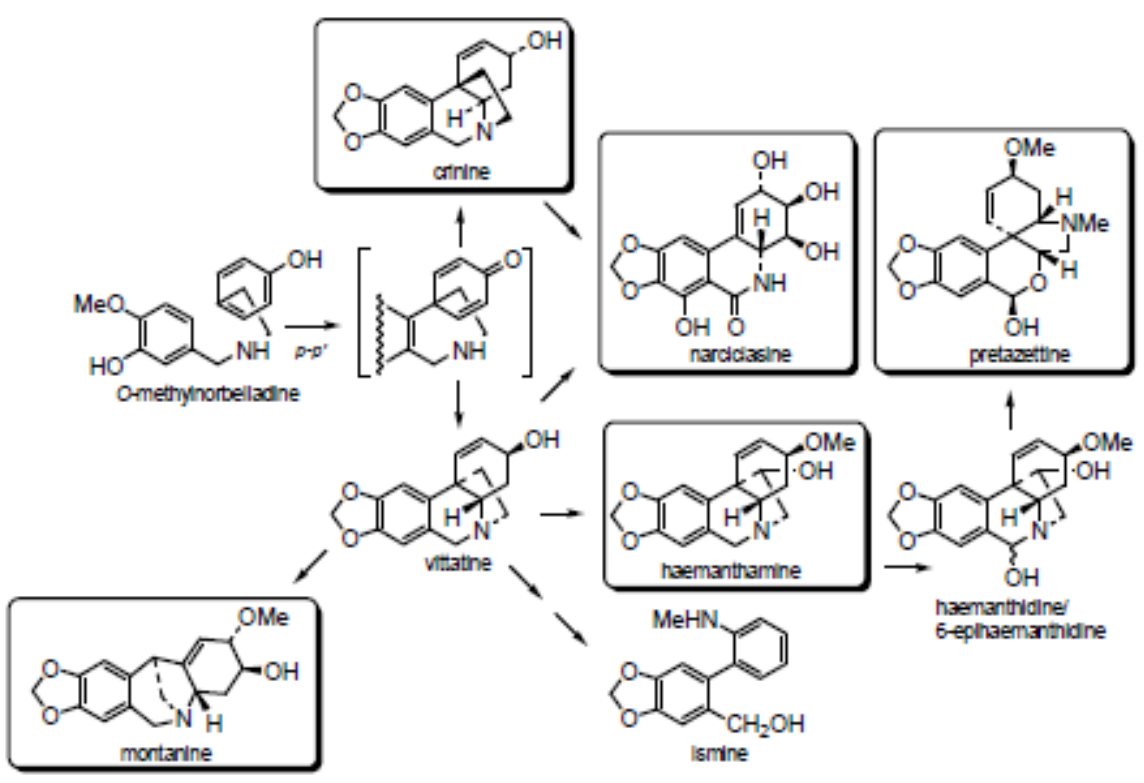

Figura 5. Alcaloides procedentes de un acoplamiento para - para'.

Fuente: Bastida et al. (2011).

Otra ruta descrita parte de la hemantamina que se forma de un acoplamiento para - para que ocurre en la estructura enantiomérica de la crinina y es el precursor de tazetina, montanina y narciclasina. Cuando la hemantamina sufre una oxidación y se transforma en hemantadina, esta experimenta una apertura del anillo por el equilibrio del grupo amino aldehído y permite la formación de un hemiacetal seguido de una metilación, dando paso a la conformación de la estructura pretazetina, que mantiene en equilibrio con tazetina debido a que es inestable tanto en medio básico como ácido (Cedrón, 2010).

\section{Formación de tipo galantamina}

Estos alcaloides tienen un núcleo dibenzofuran (tipo galantamina) y se obtienen del acoplamiento oxidativo fenol para - orto'.

Los estudios iniciales de esta vía sugirieron que el acoplamiento para - orto no procede de 0-metilnorbelladina, pero sí a partir de N,O-dimetilnorbelladina para dar finalmente galantamina (Barton et al., 1963). N,O dimetilnorbelladina se aisló de Pancratium maritimum (Vázquez-Tato et al., 1988) una especie que contiene también galantamina.

Sin embargo, el estudio más reciente parece contradecir lo anterior. Experimentos llevados a cabo con la aplicación de C13 marcado con 0-metilnorbelladina a los órganos de Leucojum aestivum muestra que la biosíntesis de galantamina implica la oxidación de fenol y el acoplamiento de O-metilnorbelladina a una dienona, que sufre cierre espontáneo del puente éter para producir $\mathrm{N}$-demetilnarwedina, dando norgalantamina después de la reducción estereoselectiva (Eichhorn et al., 1998). En contraste con la literatura, N,O dimetilnorbelladina se metaboliza en menor medida en $L$. aestivum y es incorporado en la galantamina así como norgalantamina (Figura 6 ). 


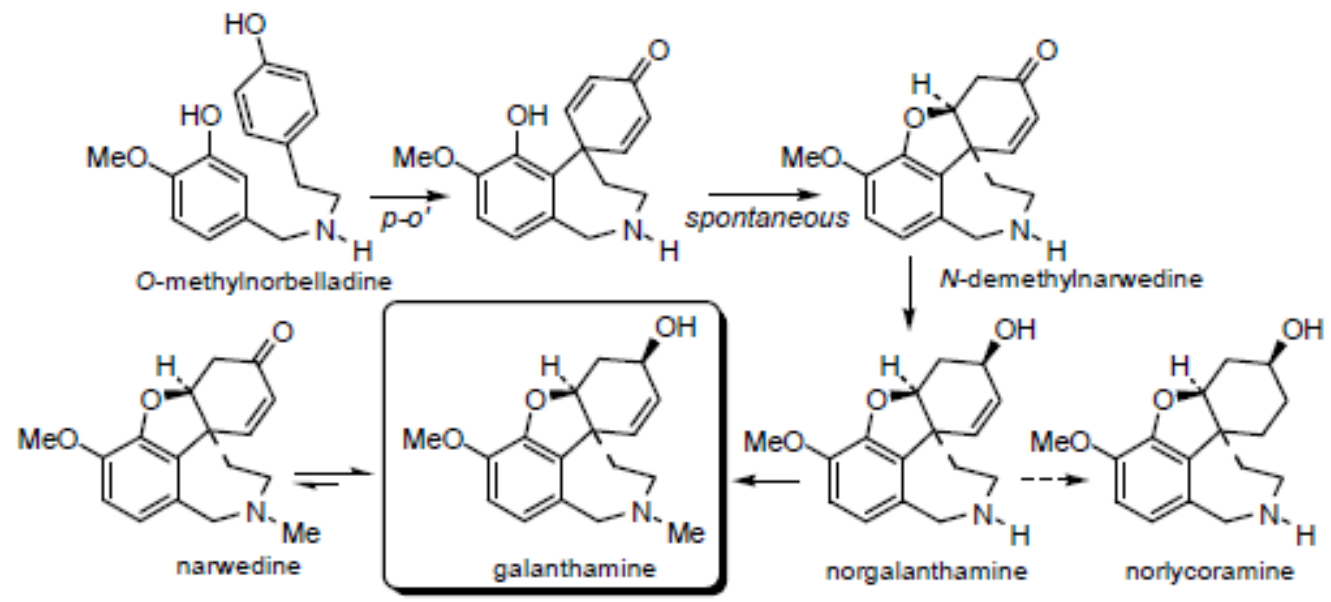

Figura 6. Biosíntesis de galantamina y derivados.

Fuente: Bastida et al. (2011).

\section{Actividades biológicas y farmacológicas}

Los alcaloides de Amaryllidaceae aplicados en la medicina tradicional poseen actividad antiviral, antimalárica, antitumoral, antimicrobiana, citotóxica, anticonvulsiva, hipotensiva, antiinflamatoria, estimulante respiratoria, entre otras propiedades. Sin embargo y hasta el momento, galantamina es uno de los alcaloides más importantes y representativos de estas plantas, ya que se ha comprobado su aplicación en el tratamiento del Alzheimer (Bastida, 2011; Ivanov et al., 2012; Konrath et al., 2013; Huaylla et al., 2020; Shammari et al, 2020).

Los alcaloides de tipo licorina han demostrado ser un potente inhibidor de ácido ascórbico (L-Asc) (Arrigoni et al., 1975), debido a la inhibición de la enzima L-galactonolactona deshidrogenasa (Kuzniak, 2004); también limita el crecimiento y división celular, además de la organogénesis en plantas superiores. Actividades semejantes fueron reportadas para diidrocolirina, psuedolicorina, haemantamina, pretazetina y narciclasina (Ghosal et al., 1985). La licorina también presenta acción analgésica mayor que el propio ácido acetilsalicílico (Fennell \& Van Staden, 2001; Nair \& Van Standen, 2019; Nair \& Van Standen, 2020a; Nair \& Van Standen, 2020b).

Para homolicorina, se ha informado sobre efectos citotóxicos contra células tumorales, como linfoma, hepatoma humano y cáncer de próstata, y no tumorales, como fibrosblásticas; también posee una alta actividad antiretroviral contra el herpes simplex tipo 1, en seres humanos (Szlávik et al., 2004; Fennell \& Van Staden, 2001; Nair \& Van Standen, 2020a; Nair \& Van Standen, 2020b). Otra característica es su actividad analgésica (Ghosal et al., 1985), antifúngica contra Candida albicans (Evidente et al., 2004) e inductora de retraso de hipersensibilidad en animales (Gude et al., 1988).

Los tipos crinina y haemantamina poseen efectos inhibidores de crecimiento celular pronunciado con una variedad de células tumorales como la leucemia, linfomas, melanomas, hepatomas y cáncer de próstata (Youssef \& Frahm, 1998; Furusawa et al., 1980) y no tumorales como células de fibroblasto (Weniger et al., 1995). El mecanismo de acción se basa en la inhibición de la síntesis de proteínas y bloqueo de la etapa de enlace peptídico en el centro de peptidil transferasa de la subunidad 605 ribosomal.

La tazetina es uno de los alcaloides más activos contra las células linfoides (Weniger et al., 1995), y se utiliza para tratar el virus de la leucemia de Rauscher (Furusawa et al., 1976). De hecho, pretazetina inhibe la actividad de la transcriptasa inversa de diversos virus (Ghosal et al., 1985; Bisama, 2017; Nair \& Van Staden, 2020a). 
La narciclasina es un alcaloide antimitótico y antitumoral (Ceriotti, 1967), afecta las células en división en la etapa de metafase e inhibe la síntesis de proteínas en ribosomas de eucariotas por la interacción directa con la subunidad $60 S$ (Rodríguez et al., 1995). Retarda la síntesis de ADN e inhibe la citotoxicidad inducida por la calprotectina (Mikami et al., 1999). En la planta es un potente inhibidor de germinación y en algunas plantas interactúa con hormonas de crecimiento (Ghosal et al., 1989). Así, el ácido indol-3-acético no puede superar la inhibición de elongación en trigo, suprime la inducción de giberelina a-amilasa para la producción de cebada y la biosíntesis de ácido ascórbico (Evidente et al., 2004; Nair \& Van Staden, 2020).

La galantamina es un alcaloide encontrado en Galanthus nivalis y en otras especies de Amaryllidaceae como Lycoris spp., Galanthus woronowi y Narcissus spp. Este alcaloide farmacológico posee actividad anticolinesterásica (Ivanov et al., 2013) y es empleado en el tratamiento de la enfermedad de Alzheimer. La terapia está centrada en la reposición del déficit de la transmisión colinérgica cortical (Puiatti et al., 2013), estimulando los receptores nicotínicos pre y postsinápticos, los cuales pueden aumentar la liberación de neurotransmisores como glutamato, estimulando la función neuronal.

Actualmente, la galantamina que se utiliza clínicamente y casi en su totalidad es producida a partir de las plantas Leucojum o Narciso (Takos \& Rook, 2013); sin embargo, su extracción a partir de hojas varía entre el 0.059 \% y 0.2 $\%$ de materia seca, por lo que su rendimiento es todavía demasiado bajo, lo que eleva su costo (Kornienko \& Evidente, 2008).

La cual tiene efectos moderadamente significativos en el alivio de los síntomas de intensidad leve y media, y muestra efectos colaterales. Otros tres fármacos que están siendo comercializados son donepezil (Aricept $®$ ), rivastigmina (Exelon ${ }^{\circledR}$ ) y galantamina (Reminyl @) (Heinrich \& Lee Teoh, 2004). Las ventajas de este último son su larga duración, selectividad, reversibilidad y competitividad, por lo que es considerado el más efectivo en el tratamiento del Alzheimer (Rhee et al., 2001).

\section{Conclusiones}

La actividad fitoquímica de la familia Amaryllidaceae muestra numerosos alcaloides con actividades biológicas y farmacológicas de importancia para gran parte de la sociedad y en especial en el tratamiento de pacientes con cáncer y con Alzheimer: se reconocen nueve armazones originados a partir de la tirosina y la fenilalanina, cuyo primer precursor es la beladina: norbelladina, licorina, homolicorina, crinina, hemantamina, narciclasina, tazetina, montanina y galantamina. Se ha documentado evidencia sobre la actividad antiviral, antimalárica, antitumoral, antimicrobiana, citotóxica, anticonvulsiva, hipotensora, antiinflamatoria y estimulante respiratoria de estos alcaloides. Destaca la galantamina, cuya actividad anticolinesterásica a partir de la liberación de neurotransmisores como glutamato, que estimulan la función neuronal, hace parte del tratamiento de la enfermedad de Alzheimer, o la homolicorina, sobre la que hay evidencia de sus efectos citotóxicos contra células tumorales y fibroblásticas, así como retroviral contra herpes simplex tipo 1, en seres humanos. 


\section{Referencias}

Antoun, M. D., Mendoza, N. T., Ríos, Y. R. \& Proctor, G. R. (1993). Cytotoxicity of Hymenocallis Expansa Alkaloids. Journal of Natural Products. 56, 1423-1425.

Arango, G. J. (2008). Alcaloides y compuestos nitrogenados. Facultad de Química Farmacéutica, Universidad de Antioquia.

Arrigoni, O., Arrigoni-Liso, R. \& Calabrese, G. (1975). Lycorine as an Inhibitor of Ascorbic Acid Biosynthesis. Nature, 256, 513-514.

Arroyo, S. C. \& Cutler, D. F. (1984). Evolutionary and Taxonomic Aspects of the Internal Morphology In the Amarillidaceae from South American and Southern Africa. Kew Bulletin. 39, 467-498.

Barton, D. H. R., Kirby, G. W., Taylor, J. B. \& Thomas, G. M. (1963). Phenol Oxidation and Biosynthesis. Part VI. The Biogenesis of Amaryllidaceae Alkaloids. J. Chem. Soc., 4545.

N. B., de Andrade, J. P., Vanessa Martínez V. \& Viladomat, F. (2011). Chemical and Biological Aspects of Amaryllidaceae Alkaloids. In D. Muňoz-Torrero (ed), Recent Advances in Pharmaceutical Sciences. Transworld Research Network.

Bisama, I.R.L. (2017). Quimiotaxonomía de Amaryllidaceae chilenas: alcaloides como marcadores taxonómicos. (Tesis de doctorado). Universidad de Concepción. Facultad de Ciencias Naturales y Oceanográficas-Programa de Doctorado en Ciencias Biológicas área Botánica. http://repositorio.udec.cl/handle/11594/2844

Bruneton, J. (1987). Eléments de Phytochimie et de Pharmacognosie. Ed. Technique et Documentation -Lavoisier. Cabezas, F., Pigni, N., Bastida, J., Codina, C., Viladomat, F. (2013). Analisis del contenido alcaloidico de Caliphruria subedentata baker (Amaryllidaceae) por el metodo CG-EM. Rev. Latinoamer. Quím. 41 (1), 68-73.

Cahlíková, L., Valterová, I., Macáková, K., \& Opletal, L. (2011). Analysis of Amaryllidaceae Alkaloids from Zephyranthes Grandiflora by GC/MS and their Cholinesterase Activity. Revista Brasileira de Farmacognosia, 21(4), 575-580.

Cedrón, M. J. A. B. (2010). The Alkaloids. In Chemistry and Biology of Pancratium Alkaloids (pp. 2-6). Elsevier.

Ceriotti, G. (1967). Narciclasine: An Antimitotic Substance from Narcissus Bulbs. Nature, 213, 595-596.

Chu, H. Y., Wegel, E., \& Osbourn, A. (2011). From Hormones to Secondary Metabolism: The Emergence of Metabolic Gene Clusters in Plants. Plant Journal, 66(1), 66-79. https://doi.org/10.1111/j.1365-313X.2011.04503.X.

Desgagne-Penix, I. \& Singh, A. (2012). Biosynthesis of the Amaryllidaceae Alkaloids. Plant Science Today 1(3), 114120. https://doi.org/10.14719/pst.2014.1.3.41

Desgagne-Penix, I., Khan, M. F., Schriemer, D. C., Cram, D., Nowak, J., \& Facchini, P. J. (2010). Integration of Deep Transcriptome and Proteome Analyses Reveals the Components of Alkaloid Metabolism In Opium Poppy Cell Cultures. BMC Plant Biology, 10, 252. https://doi.org/10.1186/1471-2229-10-252. 
Dewick, P. M. (2009). Medicinal Natural Products: A Biosynthetic Approach (3rd ed.). John Wiley and Sons. https://doi.org/10.1002/9780470742761.

Dewick, P. M. (2002). Medicinal Natural Products: A Biosynthetic Approach (2nd. Ed.). John Wiley y Sons.

Dutilh, J. H. A. (1996). Biossistemática de quatro espécies de Hippeastrum Herb. (Amaryllidaceae). (Tese de doutorado). UNICAMP (SP).

Eichhorn, J., Takada, T., Kita, Y., \& Zenk, M. H. (1998). Biosynthesis of the Amaryllidaceae Alkaloid Galanthamine. Phytochemistry, 49(4), 1037-1047. https://doi.org/10.1016/S0031-9422(97)01024-8

Elgorashi, E. E., Drewes, S. E., Morris, C. \& Staden, J. V. (2003). Variation among Three Crinum Species in Alkaloid Content. Biochemical Systematics and Ecology. 31, 601-615.

Evidente, A., Andolfi, A., Abou-Donia, A. H., Touema, S. M., Hammoda, H. M., Shawky, E. \& Motta, A. (2004). (-)Amarbellisine, a lycorine-type Alkaloid from Amaryllis Belladonna L. Growing in Egypt. Phytochemistry, 65, 21132118. https://pubmed.ncbi.nlm.nih.gov/15279981/

Facchini, P. J. (2001). Alkaloid Biosynthesis in Plants: Biochemistry, Cell Biology, Molecular Regulation, and Metabolic Engineering Applications. Annual Review of Plant Physiology and Plant Molecular Biology. 52, 29-66. https://doi.org/10.1146/annurev.arplant.52.1.29.

Facchini, P. J., Huber-Allanach, K. L., \& Tari, L. W. (2000). Plant Aromatic L-Amino Acid Decarboxylases: Evolution, Biochemistry, Regulation, and Metabolic Engineering Applications. Phytochemistry, 54(2), 121-138. https://doi.org/10.1016/S0031-9422(00)00050-9.

Fales, H. M. y Wildman, W. C. (1964). Amaryllidaceae Alkaloids and Colchicine. J. Am. Chem. Soc., 86, 105-112. Fennell C. W. y van Staden, J. (2001). Crinum Species in Traditional and Modern Medicine. J. Ethnopharmacol. 78, 15-36. https://www.elsevier.com/

Fernández, J. L., \& Groenendijk, J. P. (2004). A New Specie of Zephyranthes Herb. sl (Amaryllidaceae, Hippeastreae) with Notes on the Genus in Colombia. Rev. Acad. Colomb. Cienc. 28(107), 177-186.

Fuganti, C. \& Mazza, M. (1971). Relative Stereochemistry of Protonation and Hydroxylation in the Biosynthesis of Lycorenine and Haemanthidine from Protocatechualdehyde. J. Chem. Soc. Chem. Commun, 19, 1196-1197.

Fuganti, C., \& Mazza, M. (1973). Biosynthesis of amaryllidaceae alkaloids. Stereochemistry of hydrogen removal a to the tertiary nitrogen atom in the biological conversion of norpluviine into lycorenine. Journal of the Chemical Society, Perkin Transactions 1, 954-956.

Furusawa, E., Furusawa, S., Lee, J. Y. B. \& Patanavanich, S. (1976). Proc. Soc. Exp. Biol. Med. 152, 186.

Furusawa, E., Irie, H., Combs, D. \& Wildman, W. C. (1980). Therapeutic Activity of Pretazettine on Rauscher Leukemia: Comparison with the Related Amaryllidaceae Alkaloids. Chemotherapy, $26,36$.

Ghosal, S., Singh, S., Kumar, Y. \& Srivastava, S. (1989). Occurrence of Two Epimeric Alkaloids and Metabolism Compared with Lycorine in Crinum Latifolium. Phytochemistry, 28, 2535-2537.

Ghosal, S., Shanthy, A. \& Singh, S. K. (1988). Isocraugsodine, an n-arylidenephenethylamine from Crinum asiaticum and its e-z isomerism. Phytochemistry, 27(6), 1849-1852. https://doi.org/10.1016/0031-9422(88)80457-6. 
Ghosal, S., Saini, K. S. \& Razdan, S. (1985). Phytochemistry. 10, 2141-2156.

Grisebach, H. (1973). Comparative Biosynthetic Pathways in Higher Plants. Pure and Applied Chemistry, 34(3-4), 487-513. https://doi.org/10.1351/pac197334030487.

Gude, M., Hausen, B. M., Heitsch, H. \& König, W. A. (1988). An Investigation of the Irritant and Allergenic Properties of Daffodils (Narcissus pseudonarcissus L., Amaryllidaceae). Contact Dermatitis, 19, 1.

Hagel, J. M., \& Facchini, P. J. (2013). Benzylisoquinoline Alkaloid Metabolism: A Century of Discovery and a Brave New World. Plant and Cell Physiology. 54(5), 647-672. https://doi.org/10.1093/pcp/pct020.

Harken, R. D., Christensen, C. P. \& Wildman, W. C. (1976). Interconversions in the Pluviine-lycorenine Series. J. Org. Chem. 41, 2450-2454.

Heinrich, M., \& Lee Teoh, H. (2004). Galanthamine from Snowdrop-the Development of a Modern Drug against Alzheimer's Disease from Local Caucasian Knowledge. Journal of Ethnopharmacology. 92(2-3), 147-162. https://doi.org/10.1016/j.jep.2004.02.012.

Herrera, M. R., Machocho, A. K., Brun, R., Viladomat, F., Codina, C., \& Bastida, J. (2001). Crinane and lycorane Type Alkaloids from Zephyranthes citrina. Planta Medica, 67(2), 191-193. https://doi.org/10.1055/s-2001-11495.

Huaylla, H., Llalla, O., Torras-Claveria, L. \& Bastida, J. (2020). Alkaloid Profile in Pyrolirion Albicans Herb. (Amaryllidaceae), a Peruvian Endemic Species. South African Journal of Botany, 000, 1-5. https://doi.org/10.1016/j.sajb.2020.03.016

Ivanov, I., Georgiev, V., \& Pavlov, A. (2013). Elicitation of Galanthamine Biosynthesis by Leucojum Aestivum Liquid Shoot Cultures. Journal of Plant Physiology, 170(12), 1122-1129. https://doi.org/10.1016/j.jplph.2013.03.017

Ivanov, I., Georgiev, V., Berkov, S., \& Pavlov, A. (2012). Alkaloid Patterns in Leucojum Aestivum Shoot Culture Cultivated at Temporary Immersion Conditions. Journal of Plant Physiology, 169(2), 206-211. https://doi.org/10.1016/j.jplph.2011.09.010

Jin, Z. (2013). Amaryllidaceae and Sceletium alkaloids. Natural Product Reports. 30(6), 849-868. https://doi.org/10.1039/c3np70005d

Konrath, E. L., Passos, C. d. S., Klein-Júnior, L. C., \& Henriques, A. T. (2013). Alkaloids as a Source of Potential Anticholinesterase Inhibitors for the Treatment of Alzheimer's Disease. Journal of Pharmacy and Pharmacology.

Kornienko, A. \& Evidente, A. (2008). Chemistry, Biology, and Medicinal Potential of Narciclasine and its Congeners. Chemical Reviews, 108(6), 1982-2014. https://doi.org/10.1021/cr078198u.

Kuzniak, E. (2004). Ascorbate and Ascorbate-Dependent Enzymes in Detached Tomato Leaves under Conditions Modulating the Ascorbate Pool. Acta Physiol. Plant. 26, 327-333.

Liscombe, D. K., Louie, G. V. \& Noel, J. P. (2012). Architectures, Mechanisms and Molecular Evolution of Natural Product Methyltransferases. Natural Product Reports. 29(10), 1238-1250. https://doi.org/10.1039/c2np20029e.

Meerow, A. W. (2004). Amaryllidaceae (Amaryllis family). Flowering Plants of the Neotropics. Princeton and Oxford. 
Mikami, M., Kitahara, M., Kitano, M., Ariki, Y., Mimaki, Y., Sashida, Y., Yamazaki, M. \& Yui, S. (1999). Biol. Pharm. Bull. 22, 674.

Nair, J. J., \& Van Staden, J. (2013). Pharmacological and Toxicological Insights to the South African Amaryllidaceae. Food Chemistry and Toxicology, 62C, 262-275. https://doi.org/10.1016/j.fct.2013.08.042

Nair, J.J \& Van Staden, J. (2019). Antiplasmodial Constituents in the Minor Alkaloid Groups of the Amaryllidaceae. South African Journal of Botany 126, 362-370. https://doi.org/10.1016/j.sajb.2019.06.012

Nair, J.J. \& Van Staden, J. (2020a). Cytotoxic Tazettine Alkaloids of the Plant Family Amaryllidaceae. South African Journal of Botany, 000, 1-10. https://doi.org/10.1016/j.sajb.2020.07.002

Nair, J.J \& Van Staden, J. (2020b). The Plant Family Amaryllidaceae as a Source of Cytotoxic Homolycorine Alkaloid Principles. South African Journal of Botany 000, 1-18. https://doi.org/10.1016/j.sajb.2020.07.013

Osorio, D. J. (2004-2006). Búsqueda de sustancias bioactivas a partir de dos especies de la flora colombiana: alcaloides de Phaedranassa dubuia (Amaryllidaceae) y Biflavonoides de Garcinia madurno (Clusiaceae). Javier Osorio Durango.

Paris, M. \& Hurabielle, M. (1981). Abrégé de Matière Médicale - Pharmacognosie. tomos 1 y 2. Masson.

Pinto, A. C., Silva, D. H. S., Bolzani, V. S., Lopes, N. P. \& Epifanio, R. A. (2002). Productos naturais: atualidade, desafios e perspectivas. Química Nova. 25, 45-61.

Puiatti, M., Borioni, J. L., Vallejo, M.G., Cabrera, J. L., Agnese, M.A., Ortega, M. G. \& Pierini, A.B. (2013). Study of the Interaction of Huperzia saururus Lycopodium Alkaloids with the Acetylcholinesterase Enzyme. Journal of Molecular Graphics and Modelling. 44,136-144.

Reyes-Chilpa, R., Berkov, S., Hernández-Ortega, S., Jankowski, C. K., Arseneau, S., Clotet-Codina, I. \& Bastida, J. (2011). Acetylcholinesterase-inhibiting Alkaloids from Zephyranthes Concolor. Molecules, 16(11), 9520-9533. https://doi.org/10.3390/molecules16119520.

Rivero, N., Gómez, M. \& Medina, J. D. (2004). Search for Bioactive Alkaloids in Hymenocallis Species. Pharmaceutical Biology, 42, 280-285.

Rhee, I. K., Van de Meent, M., Ingkaninam, K. \& Verpoorte, R. (2001). Screening for Acetylcholinesterase Inhibitors from Amaryllidaceae using Silica Gel Thin-Layer Chromatography in Combination with Bioactivity Staining. Journal of Chromatography A. 915, 217-223.

Rojas-Vera, J.C., Buitrago-Díaz, A.A., Possamai, L.M., Timmers, L.F.S.M., Tallini, L.R. \& Bastida, J. (2020). Alkaloid Profile and Cholinesterase Inhibition Activity of Five Species of Amaryllidaceae Family Collected from Mérida stateVenezuela. South African Journal of Botany 000, 1-11. https://doi.org/10.1016/j.sajb.2020.03.001

Rodríguez-Fonseca, C., Amils, R. \& Garret, R. A. (1995). Fine Structure of the Peptidyl Transferase Centre on 23 Slike rRNAs Deduced from Chemical Probing of Antibiotic-Ribosome Complexes. J. Mol. Biol. 247, 224.

Shammari, L.Al., Hulcová, D., Marikova, J., Kucera, T., Safratova, M., Nováková, L., Schmitdt, M., Pulkráková, L., Janousek, J., Soukup, O., Kunes, J., Opletal, L. \& Cahlíková, L. (2020). Amaryllidaceae Alkaloids from Hippeastrum X 
Hybridum CV. Ferrari, and Preparation of Vittatine Derivatives as Potential Ligands for Alzheimers Disease. South African Journal of Botany 000, 1-10. https://doi.org/10.1016/j.sajb.2020.06.024

Singh, A. \& Desgagné-Penix, I. (2014). Biosynthesis of the Amaryllidaceae Alkaloids. Plant Science Today, 1(3), 114120. http://dx.doi.org/10.14719/pst.2014.1.3.41

Sousa da Silva, M. S. (2009). Alcalóides de Plantas da Família Amaryllidaceae: Isolamento Caracterização e Testes de Inibição de Acetilcolinesterase. (Tese de doutorado). Universidade de Campinas (SP).

Szlávik, L., Gyuris, A., Minárovits, J., Forgo, P., Molnár, J. \& Hohmann, J. (2004). Alkaloids from Leucojum Vernum and Antiretroviral Activity of Amaryllidaceae alkaloids. Planta Med. 70, 871.

Takos, A. M., \& Rook, F. (2013). Towards a Molecular Understanding of the Biosynthesis of Amaryllidaceae Alkaloids in Support of their Expanding Medical Use. International Journal of Molecular Science, 14(6), 11713-11741. https://doi.org/10.3390/ijms140611713.

Tram, N. T. N., Titorenkova, T. V., Bankova, V. St., Handjieva, N. V. \& Popov, S. S. (2002). Crinum L. (Amaryllidaceae). Fitoterapia. 73, 183-208.

Unver, N. (2007). New Skeletons and New Concepts in Amaryllidaceae Alkaloids. Phytochemistry Review. 6, 125135.

Vázquez-Tato, M. P., Castedo, L. \& Riguera, R. (1988). New Alkaloids from Pancratium maritimum. Heterocycles, 27, 2833-2838.

Vélez-Terranova, M., Campos-Gaona, R. \& Sánchez-Guerrero, H. (2014). Uso de metabolitos secundarios de las plantas para reducir la metanogénesis ruminal. Tropical and Subtropical Agroecosystems, 17(3), 489-499. https://www.redalyc.org/pdf/939/93935728004.pdf

Weniger, B., Italiano, L., Beck, J. P., Bastida, J., Bergoñon, S., Codina, C., Lobstein, A. \& Anton, R. (1995). Cytotoxic Activity of Amaryllidaceae Alkaloids. Planta Med. 61, 77.

Wildman, W. C. \& Bailey, D. T. (1969). Amaryllidaceae Interconversions. Partial Syntheses of [2]benzopyrano[3,4c]indoles. J. Am. Chem. Soc. 9, 150-157.

Youssef, D. T. A. \& Frahm, A. W. (1998). Alkaloids of the Flowers of Pancratium Maritimum. Planta Med. 64, 669.

Ziegler, J. \& Facchini, P. J. (2008). Alkaloid Biosynthesis: Metabolism and Trafficking. Annual Review of Plant Biology, 59, 735-769. https://doi.org/10.1146/annurev.arplant.59.032607.092730.

Cómo citar este artículo: González-Chavarro, C., Cabezas, M., Pulido, V. \& Celis, X. (2020). Amaryllidaceae: fuente potencial de alcaloides. Actividades biológicas y farmacológicas. Cien. Agri. 17(3), 78-94. https://doi.org/10.19053/01228420.v17.n3.2020.11379 